\title{
LES Predictions of Self-Sustained Oscillations in Homogeneous Density Round Free Jet
}

\author{
K. Wawrzak ${ }^{1}$ (D) A. Boguslawski ${ }^{1}$ - A. Tyliszczak ${ }^{1}$
}

Received: 5 January 2015 / Accepted: 6 August 2015 / Published online: 21 August 2015

(C) The Author(s) 2015. This article is published with open access at Springerlink.com

\begin{abstract}
The paper presents a detailed LES analysis of turbulent round jets dominated by the mechanism of Kelvin-Helmholtz $(\mathrm{K}-\mathrm{H})$ instability and the so-called self-sustained regime, which is characterised by large velocity fluctuations, reminiscent of the behaviour of excited jets. It is shown that the occurrence of this regime is largely conditioned by the type and parameters of the inlet jet velocity profile, i.e., the shear layer momentum thickness $\theta$, turbulence intensity $T i$. A high order numerical code based on the combined pseudospectral / compact difference methods is used in the simulations. Analysis is performed for the Reynolds number $R e=1 \times 10^{4}$ with $\theta$ characterised by $R / \theta=16,20,24,28$ and 32 (with $R$ - jet radius) and for $T i=10^{-2}, 10^{-3}, 10^{-4}$. Two inlet velocity profiles are used in the simulations: hyperbolic tangent and Blasius. Comparisons focus on the axial velocity profiles and the spectra of the velocity signals. It is shown that in the self-sustained regime the results obtained with the Blasius profile are significantly closer to the experimental data. Sensitivity tests of the self-sustained regime on the sub-grid modelling are performed based on four well known models: classical and dynamic Smagorinsky, the filtered structure function model of Ducros et al. (JFM, 1996) and the relatively new model proposed by Vreman (PoF, 2004). It is shown that in the case of the classical Smagorinsky model an excess of sub-grid dissipation prevents the appearance of self-sustained velocity oscillations and in effect gives results significantly different from the remaining models. On the other hand, when the jets are dominated by K-H instability all the models lead to very similar solutions.
\end{abstract}

Keywords Self-sustained oscillations · LES predictions · Round jet instability · Kelvin-Helmholtz instability

K. Wawrzak

wawrzak@imc.pcz.czest.pl

1 Institute of Thermal Machinery, Czestochowa University of Technology,

Al. Armii Krajowej 21, 42-200 Czestochowa, Poland 


\section{Introduction}

Large scale coherent structures in the near field of round free jets have been widely studied by experimental and numerical investigations since the pioneering experiments of Crow \& Champagne [1] and Brown \& Roshko [2]. However, as it was pointed out in a recent review paper by Ball et al. [3] on the flow field in turbulent round free jets, despite the great attention that the round jet received in the last century there remain some significant outstanding questions. The first one stressed by Ball et al. [3] was the route or mechanism leading to self-similarity in the round free jet. It seems that any understanding of this mechanism still requires a new insight into the dynamics of large scale highly anisotropic vortex structures formed within the first few diameters of the nozzle. Boguslawski et al. [6], in a recent work on a jet with a very low turbulence level at the nozzle exit, observed unusually high oscillations in the homogeneous density natural round jet, comparable to those observed in externally stimulated flows as in the experiment of Crow \& Champagne [1]. This experimental observation was a motivation for intense numerical simulations of this flow type. Using LES predictions Boguslawski et al. [6] showed that these high flow field oscillations are triggered by a self-sustained mechanism of a fully convective nature. It was found that the occurrence of a regime with self-sustained oscillations requires a relatively low turbulence level and a sufficiently thin shear layer at the nozzle exit. Under such conditions large scale coherent vortices develop in the space fast enough to generate an external back flow stimulating flow oscillations close to the nozzle exit. In order to shorten the notation, this regime is called the self-sustained regime hereafter in this paper. The critical shear layer thickness characterized by the parameter $R / \theta \approx 25$ ( $R$-nozzle radius, $\theta$-momentum thickness of the shear layer at the nozzle exit) was established based on the LES predictions. It is worth noting here that contamination of the inflow region by free vortical structures in the outer flow for $R / \theta>25$ was also observed by Lesshafft et al. [7] in their DNS predictions of globally unstable round jets. However, the authors did not study this effect further, limiting their attention to the shear layer thicker than the critical one reported by Boguslawski et al. [6]. Moreover, in the recent experimental work of Mi et al. [8], hot-wire measurements of velocity fluctuations along the jet centreline revealed very high turbulence intensity at a level of $16-18 \%$ and a distance $2-3$ nozzle diameters, resembling the experimental and numerical results of Boguslawski et al. [6]. These high level oscillations were observed for a range of the Reynolds number $R e=5000-8000$, for which $R / \theta=30-40$ i.e. higher than the critical value. For the higher Reynolds number this distinct peak on the fluctuating velocity profile disappeared despite $R / \theta>40$, which needs further investigation. The authors do not discuss these effects in detail, focusing on the far field jet structure instead.

Boguslawski et al. [6], using experimental and numerical results, revealed an overall mechanism leading to self-sustained oscillations, pointing out some discrepancies between experimental and numerical results. The present study was mainly aimed at explaining the reasons behind the discrepancies reported in [6]. The first problem taken into consideration was the influence of the inlet velocity profile on the flow field in the self-sustained regime. In previous numerical simulations the commonly used hyperbolic-tangent velocity profile was applied at the nozzle exit. However, in this experiment the laminar shear layer described by the Blasius velocity profile was reported. Hence, in the first part of the paper, differences of jet development with two different inlet velocity profiles are analysed.

As pointed out by Meneveau and Katz [9], an important question that has so far received little attention is how the sub-grid stress model affects the coherent structures. It was already shown by Vreman et al. [27] that different models lead to qualitative differences in the 
coherent structures of the mixing layer. The instabilities and the roll-up of vortices pose special challenges for sub-grid modeling since the initially small-scale vortices within the shear layer interact to create substantially larger structures. It seems that recently found self-sustained oscillations in the homogeneous density round jet is a new challenge for subgrid models especially during the transition from the Kelvin-Helmholtz to the self-sustained regime. Hence, in the second part the influence of the sub-grid model on jet instability development in the self-sustained regime is investigated.

\section{Modelling and Numerical Algorithm}

For incompressible, constant density flows the continuity and the Navier-Stokes equations in the framework of LES are given as [13-15] :

$$
\begin{gathered}
\frac{\partial \bar{U}_{i}}{\partial x_{i}}=0 \\
\frac{\partial \bar{U}_{i}}{\partial t}+\frac{\partial \bar{U}_{i} \bar{U}_{j}}{\partial x_{j}}=\frac{1}{\rho} \frac{\partial \bar{P}}{\partial x_{i}}+\frac{\partial}{\partial x_{j}}\left[\left(v+v_{s g s}\right)\left(\frac{\partial \bar{U}_{i}}{\partial x_{j}}+\frac{\partial \bar{U}_{j}}{\partial x_{i}}\right)\right]
\end{gathered}
$$

where the bar symbol represents the spatial filtering [13]. The variables $\bar{U}_{i}, \bar{P}, \rho$ stand for the velocity components, pressure and density, respectively. The symbols $v, v_{s g s}$ are the kinematic viscosity and sub-grid viscosity. In the present work we compare the results obtained after applying four well known sub-grid models: (i) the filtered structure function model proposed by Ducros et al. [16]; (ii) the dynamic Smagorinsky model [28], commonly referred to as the Germano model; (iii) the model proposed by Vreman [29]; (iv) the original Smagorinsky model [30]. The most important difference between these models seems to be that in the first three cases the sub-grid viscosity vanishes in laminar flows or pure shear regions while the Smagorinsky model always leads to a non-zero sub-grid viscosity, unless the velocity gradients are zero. We will examine the role of these sub-grid models in jet flows with low and medium turbulent intensity at the inlet and for jets in the self-sustained regime.

\subsection{Computational domain and boundary conditions}

A schematic view of the experimental configuration studied in [6], to which we refer in the present paper, is shown in Fig. 1. A jet of diameter $D$ issues from the nozzle with an external size equal to $3 D$. The inner shape of the nozzle is prescribed by a cubic function resulting in a contraction 12:1. In the simulations performed in the present work we simplify the problem and we do not include the region of the nozzle. Instead, we model the jet through an instantaneous velocity profile. The computational domain is a rectangular box $10 D \times 10 D \times 18 D$ with the inlet boundary defined on the bottom side. At the lateral boundaries we assume periodic boundary conditions. The size of the computational domain in the periodic directions $(10 D \times 10 D)$ was found to be sufficiently large to minimize the influence of the periodic boundary conditions on the jet flow. This was verified in test computations using a wider domain $(12 D \times 12 D)$.

The inlet boundary conditions are specified in terms of instantaneous velocity as $U(\mathbf{x}, t)=U_{\text {mean }}(\mathbf{x})+u(\mathbf{x}, t)$, where $U_{\text {mean }}(\mathbf{x})$ is the mean velocity and $u(\mathbf{x}, t)$ represents turbulent fluctuations computed according to the algorithm proposed by Kempf et al. [18] 


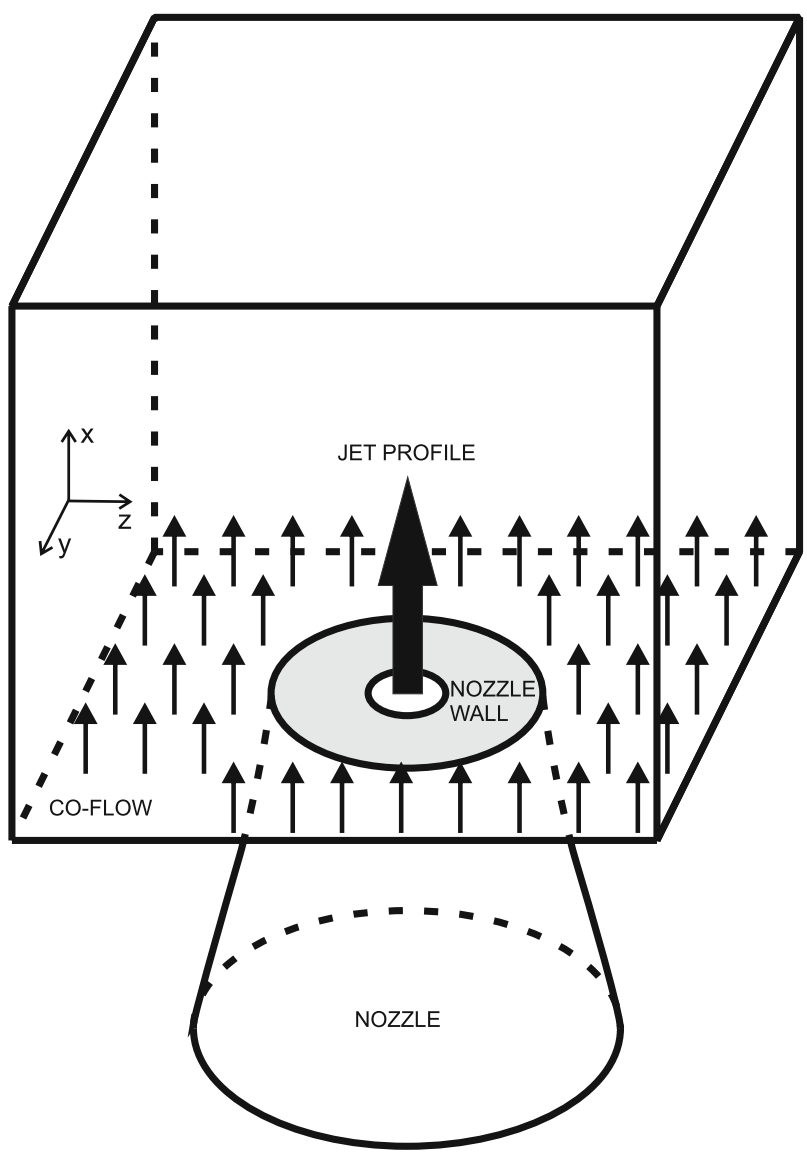

Fig. 1 Schematic view of the analyzed configuration. The computational domain is the rectangular box

which creates reliable turbulent conditions with prescribed Reynolds stresses and length scales. The pressure at the inlet is computed from the Neumann condition $\mathbf{n} \cdot \nabla p=0$ with $\mathbf{n}$ the outward normal vector. As mentioned in the introduction to this paper we focus mainly on the near field behaviour of the jet. It is expected that the results may significantly depend on the mean velocity profile. Particularly in those cases with low turbulence intensities, as in [6], where the self-sustained regime is observed. Hence, in this paper, we will compare the results obtained using two velocity profiles at the inlet: the hyperbolic-tangent profile and the Blasius profile. Although the hyperbolic-tangent profile is commonly used in jet flow simulations it is worth noting that such a profile is not one which can be measured at the nozzle exit. It is rather reminiscent of the velocity profile formed downstream of the nozzle exit. From this point of view, the Blasius profile better compares to measurements (Cf. Fig. 2b). We will show how this subtle difference influences the results in the self-sustained regime.

The hyperbolic-tangent profile is defined as:

$$
U_{\text {mean }}(\mathbf{x})=\frac{U_{0}+U_{\infty}}{2}-\frac{U_{0}+U_{\infty}}{2} \tanh \left[\frac{1}{4} \frac{R}{\theta}\left(\frac{r(\mathbf{x})}{R}-\frac{R}{r(\mathbf{x})}\right)\right]
$$




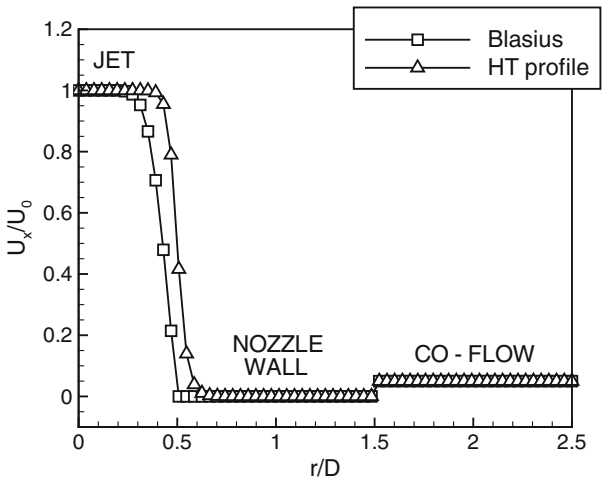

(a)

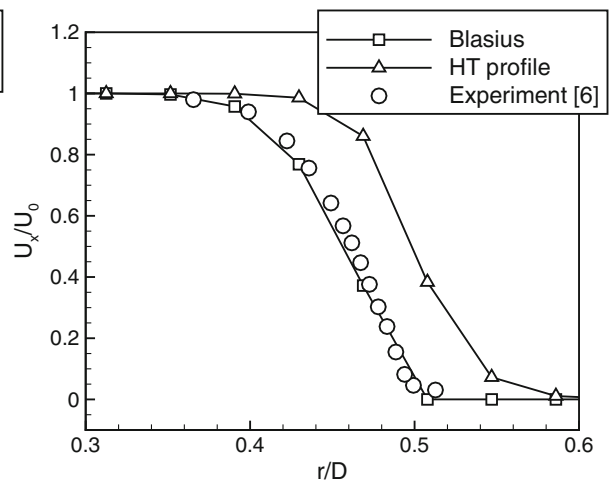

(b)

Fig. 2 Comparison of the hyperbolic tangent and Blasius profiles for (a) $R / \theta=20$, (b) $R / \theta=28$. The symbols on the lines indicate the locations of the grid nodes

where $U_{0}$ and $U_{\infty}$ denote the jet centerline velocity and the co-flow velocity and the symbol $r=\sqrt{y^{2}+z^{2}}$ is the jet radius. The co-flow is introduced to compensate for the lack of entrainment flow through the periodic lateral boundaries and natural suction through the inlet plane. In the experimental conditions of Ref. [6] the jet issued from a nozzle with a very thick wall extending over $0.5 D \leq r \leq 1.5 D$ as is shown in Fig. 1. In this region we assume $U_{\infty}=0$ and for $r>1.5 D$ we assume $U_{\infty}=0.05 U_{0}$. It was shown in [17] that the co-flow at this level does not alter the jet dynamics.

The Blasius velocity profile is obtained by solving the Blasius equation by the power series method which when combined with the co-flow velocity leads to the following formula:

$$
U_{\text {mean }}(\mathbf{x})= \begin{cases}\frac{U_{0}}{2} \sum_{n=1}^{\infty}(-1)^{n} \frac{p_{n}}{(n-1) !}\left(\frac{1}{2} r(\mathbf{x}) \frac{0.664}{\theta}\right)^{n-1} & \text { for } r(\mathbf{x}) \leq 0.5 D \\ U_{\infty} & \text { for } r(\mathbf{x})>0.5 D\end{cases}
$$

where:

$$
p_{n}=\sum_{i=0}^{n-3}\left(\begin{array}{c}
n-3 \\
i
\end{array}\right) p_{i} p_{n-1-i}, n \geq 3, p_{0}=0.6499, p_{1}=-1.2594, p_{2}=1.0668
$$

In the simulations we use Cartesian meshes on which the round shape of the jet can be represented only approximately. The edge of the jet has a stepped profile conforming to the distribution of the nodes for which the condition $\sqrt{y^{2}+z^{2}} \leq R$ holds. It becomes more accurate, i.e. closer to a circle, with an increasing number of nodes. As will be shown later, the mesh density does not affect the results significantly, and hence, we assume that the shape of the jet at the inlet reflects the real round jet sufficiently well. Radial distributions of the velocity computed from Eq. (3) and Eq. (4) are presented in Fig. 2 showing a comparison between the hyperbolic tangent (referred to from here on as HT) and Blasius profiles. It can be readily seen that in the case of the HT profile the velocity at the edge of the nozzle $(r / D=0.5)$ is equal to 0.5 whereas it is zero for the Blasius profile. Hence, applying the Blasius velocity profile will result in a slightly smaller flow rate. It is very important to note that the HT profile has an inflexion point at $r / D=0.5$ while, in the case of the Blasius profile, the inflexion point is not present at all. As will be shown in the paper this difference leads to significantly different flow behaviour. 
At the outlet plane all the velocity components are computed from a convective boundary condition $\partial U_{i} / \partial t+U_{C} \partial U_{i} / \partial n=0$ with $U_{C}$ the convection velocity computed every time step as the mean velocity in the outlet plane, limited such that $U_{C}=\max \left(U_{C}, 0\right)$. The pressure is assumed to be constant at the outflow.

\subsection{Numerical method}

The set of Eqs. (1-2) is advanced in time with a low-storage 3rd order of accuracy three-step Runge-Kutta method [19]. Within each sub-step the projection method [11] is applied for the pressure-velocity coupling. The spatial discretization of the continuity and the NavierStokes equations is performed using a combined high-order compact difference method [20] and pseudo-spectral method [21]. The former is applied in the direction of the jet axis and the latter in the periodic directions. In the case of the compact method both the first and second derivatives are discretized with a 6 th order scheme for the central nodes and 4th order boundary scheme [22]. In the case of the pseudo-spectral method the calculations of the convective terms are complemented by a dealiasing procedure according to the $3 / 2$ law [21]. The numerical code used in the present study was used previously in simulations of constant and variable density jet flows [6, 23], channel flows [24] and two-phase flows [25].

\section{Results}

The computations are performed for Reynolds number $\operatorname{Re}=U_{0} D / v=1 \times$ $10^{4}$. We compare the results obtained for jets having various momentum thicknesses $R / \theta=16,20,24,28$ and 32 and various inlet turbulent intensities $T i=u^{\prime} / U_{0}=$ $10^{-4}, 10^{-3}$ and $10^{-2}$. It is worth mentioning that in the case of the HT profile the normalized bulk velocity is equal to $1.026,1.016,1.011,1.008,1.006$ for $R / \theta=$ $16,20,24,28,32$ respectively. Hence, it is seen that a variation of $R / \theta$-parameter affects the flow rate insignificantly and the bulk Reynolds number is equal to about $1 \times 10^{4}$ for all boundary layer thicknesses. By contrast, when the Blasius profile is applied the normalized bulk velocity increases along with the reduction in boundary layer thickness and is equal to $0.766,0.828$ for $R / \theta=20,28$, respectively. In these cases changes in the bulk Reynolds numbers are more significant, but it seems that the proper velocity scale that characterizes the flow dynamics in the near jet field is the jet velocity at the nozzle axis. Hence, we expect that this change does not play an important role. Preliminary studies were carried out in order to assess the dependence of the results on the mesh density. Comparative computations were performed on uniform meshes consisting of $256 \times 256 \times 180$ and $384 \times 384 \times 180$ nodes. As will be shown below, the results obtained on both meshes are very similar. Figure $2 \mathrm{a}$ shows the locations of the grid nodes across the jet region and in the shear layer region on the coarser mesh for velocity profiles with $R / \theta=20$. In this case the formulas (3) and (4) yield a shear layer thicknesses $\delta_{99} \approx 0.48 R$ and $\delta_{99} \approx 0.375 R$, respectively. The parameter $\delta_{99}$ is defined here as the region where $U_{\infty}<U_{\text {mean }}(\mathbf{x}) \leq 0.99 U_{0}$. On the coarser mesh the number of nodes across $\delta_{99}$ is $9,7(6), 6,5(4)$ and 4 respectively for $R / \theta=16,20,24,28$ and 32. The values in brackets correspond to the number of nodes across $\delta_{99}$ in the case of the Blasius profile. The test computations on the denser mesh were performed with the HT inlet profile, with $T i=10^{-4}$ and with the shear layer characterised by $R / \theta=20$ and $R / \theta=32$. For these two cases the number of nodes across $\delta_{99}$ was equal 
to 10 and 6, respectively. From the point of view of necessary mesh resolution the computations with a very low $T i$ level require the most refined mesh, particularly for the case with $R / \theta=32$. Hence, one may assume that the analysis of the influence of the mesh density performed for the selected test cases is also meaningful for other cases with larger $T i$ levels.

Figure 3 shows profiles of the time-averaged axial velocity and its fluctuations along the jet axis. The time-averaged results were obtained by averaging the solutions over the time period $300 \mathrm{D} / U_{0}$ which corresponds to 12.5 of the so-called 'flow through time' and which was found to be sufficiently long enough to obtain convergent statistics. It can be seen in Fig. 3 that the results obtained on the coarser and denser meshes are in good agreement. For $R / \theta=20$ the length of the potential core is equal to $5 D$ on both the meshes. The decay of velocity downstream of the potential core is also very similar. Small discrepancies are observed only when comparing the profiles of the fluctuations. On the coarser mesh the occurrence of the maximum of fluctuations is predicted at $x / D=7$ which is approximately $1 D$ closer than on the denser mesh. However, the maximum level of fluctuations is the same on both meshes. The results obtained for $R / \theta=32$ also show that the coarser mesh ensures accurate results. Shortening of the potential core is reflected exactly the same on both meshes. As will be discussed in the next section, for $R / \theta=32$ we observe the self-sustained regime characterised by the occurrence of two maxima in the profiles of the fluctuations. It can be seen that this phenomenon is also well predicted on both the meshes, the only difference being that the maxima of the fluctuations on the coarser mesh are slightly larger. Based on the presented comparisons we assume that the coarser mesh with $256 \times 256 \times 180$ nodes provides reliable results. Hence, all the results presented in the next sections were obtained on this mesh.
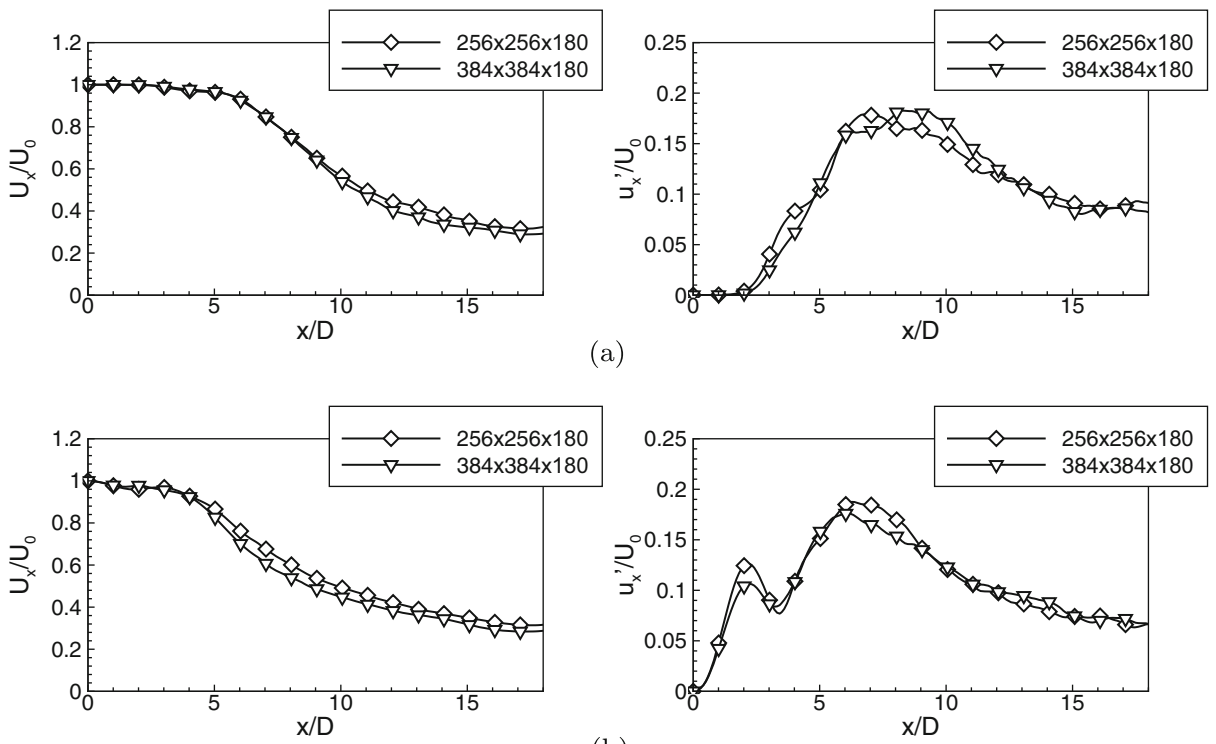

(b)

Fig. 3 Mean and fluctuating axial velocity profiles along the jet axis for $T i=10^{-4}$, (a) $R / \theta=20,(\mathbf{b})$ $R / \theta=32$. Comparison of results obtained for various computational meshes 


\subsection{Self-sustained regime}

In this section we concentrate on a demonstration of the self-sustained regime observed in [6]. The presented results are obtained with the HT inlet velocity profile and with the filtered structure function model for sub-grid scales. Comparison of the results obtained using the HT and Blasius profiles and an analysis of the sub-grid models will be presented in Section 3.2 and Section 3.5, respectively.

Figure 4 shows the profiles of the time-averaged axial velocity and its fluctuations along the jet axis obtained for the case with $R / \theta=20$ and $T i=10^{-4}, 10^{-3}$ and $10^{-2}$. In Fig. 4 it can be seen that both the mean and fluctuating velocity profiles reveal features characteristic of a round free jet with a potential core length equal to $x / D=3-5$ and maximum fluctuations at a level of $15 \%$ occurring at a distance from the inlet $x / D=6-7$ depending on $T i$. It is seen that for cases with $T i=10^{-2}$ the potential core is shorter and the velocity downstream of the potential core decays faster compared to cases with $T i=10^{-3}$ and $T i=10^{-4}$. For $T i=10^{-2}$ a sudden increase in the fluctuations begins closest to the inlet and their maximum is reached also closest to the inlet, already at $x / D=6$. Moreover, compared to the remaining cases it can be seen that for $T i=10^{-2}$ maximum fluctuations is the largest. This rather expected behaviour changes drastically when the jets attain a self-sustained regime characterized by strong velocity oscillations appearing near the inlet. As was shown in [6] the necessary condition to trigger these oscillations is that the inlet

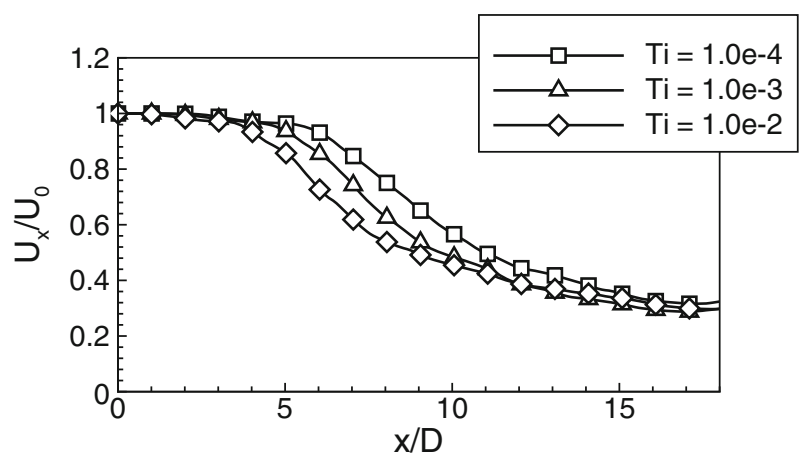

(a)

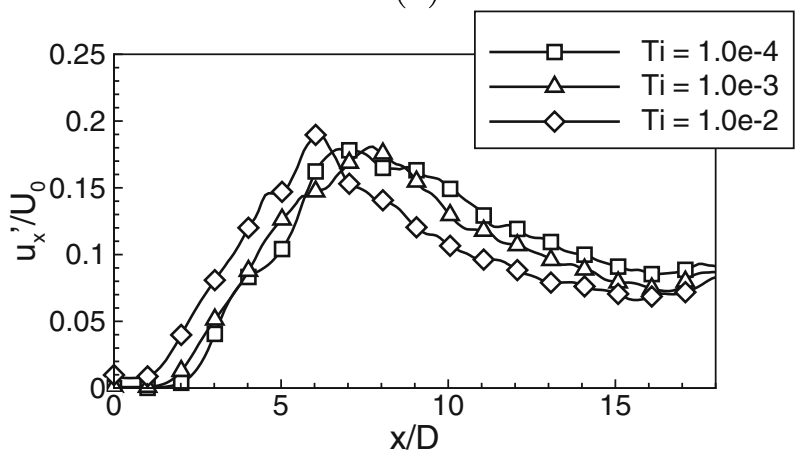

(b)

Fig. 4 Profiles of the time-averaged axial velocity (a) and its fluctuations (b) along the jet axis. Results obtained with $\mathrm{HT}$ velocity profile with $R / \theta=20$ 
momentum thickness of the jet must be sufficiently thin and the $T i$ level must be relatively low. These conditions are met in the simulations with $R / \theta=28$ for which the time-averaged mean and fluctuating velocity profiles are presented in Fig. 5. In the zoomed region of the potential core in Fig. 5a it can be seen that the mean velocity profile has a local minimum at a distance of $x / D=2-3$. This corresponds exactly to the location of the local maximum of the fluctuating velocity profile shown in Fig. 5b. We should remember that the occurrence of such local maxima of the fluctuations was first reported by Crow and Champagne [1] as the result of acoustic upstream excitation. Here, the mechanism that induces this effect is related to the self-sustained oscillations caused by feedback forcing originating from the very strong toroidal vortices generated in the shear layer region [6]. It was found that these vortices create an external back flow that acts as a hydrodynamic feedback loop and imposes disturbances on the flow right after the inlet plane. In this view such disturbances mimic external excitation and lead to results qualitatively similar to those observed by Crow and Champagne [1]. In the self-sustained regime the fastest growth in perturbation occurs for the lowest $T i$ level as can be seen in Fig. $5 b$.

Figure 6 shows the evolution of the mean and fluctuating velocities as a function of the momentum thickness for selected value of turbulence intensity $T i=10^{-4}$. It can be seen that the ratio $R / \theta$ has a very large impact on the results. It is shown that for increasing $R / \theta$ (i.e. when the shear layer thickness becomes thinner) the perturbation grows faster and when $R / \theta$ reaches some critical value the self-sustained regime is triggered. Here, this regime is

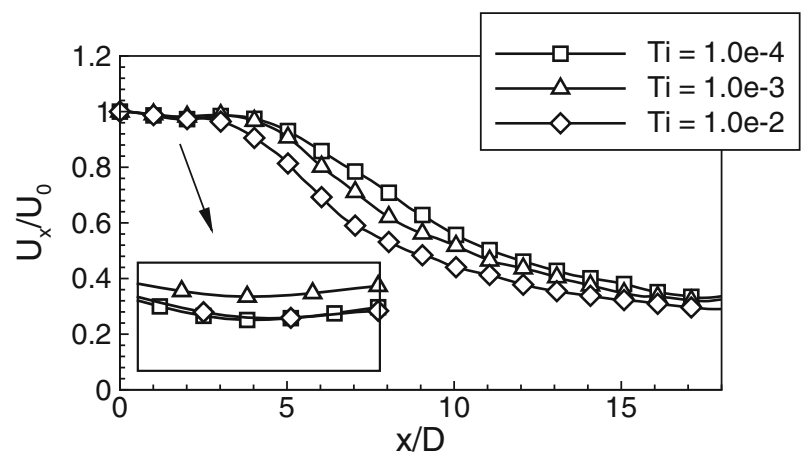

(a)

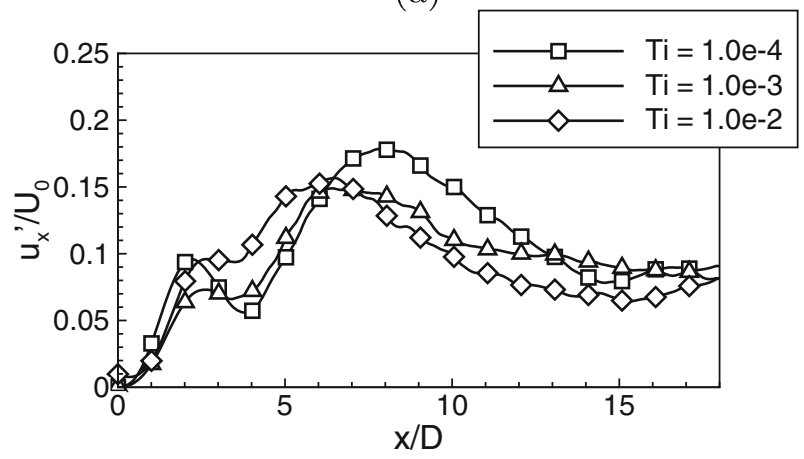

(b)

Fig. 5 Profiles of the time-averaged axial velocity (a) and its fluctuations (b) along the jet axis. Results obtained with HT velocity profile with $R / \theta=28$ 


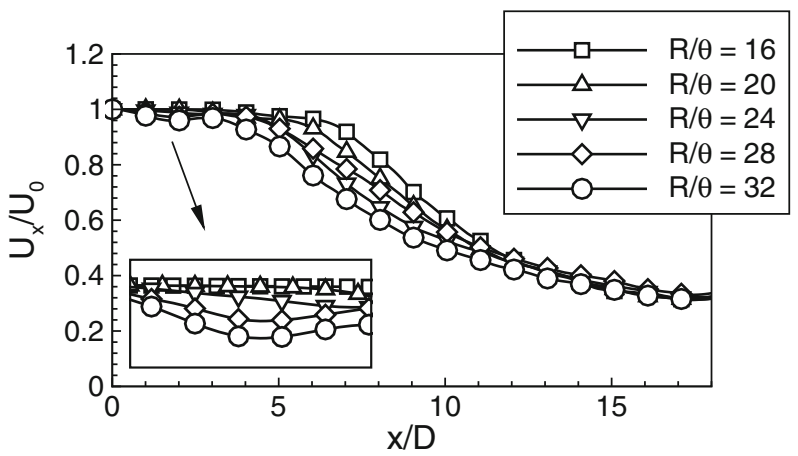

(a)

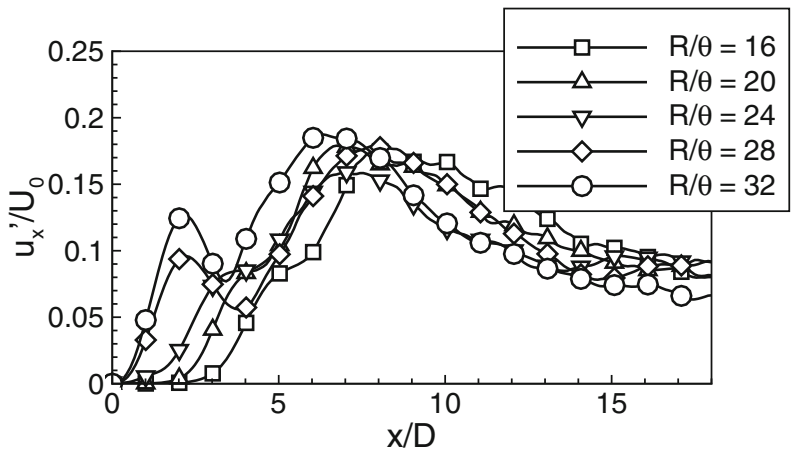

(b)

Fig. 6 Profiles of the time-averaged axial velocity (a) and its fluctuation (b) along the jet axis. Results obtained with HT velocity profile with $T i=10^{-4}$ and various $R / \theta$

observed for $R / \theta=28$ and $R / \theta=32$. In [6] the critical value $R / \theta$ for $\operatorname{Re}=1 \times 10^{4}$ was estimated as $R / \theta_{\text {crit }}=25$. Additionally, it was found that the locations of the maxima of fluctuations and minima of the mean profiles are independent of $R / \theta$ and appear at a distance $x / D \approx 2$. On the other hand the height of these extrema increases with $R / \theta$, as is confirmed in Fig. 6 b.

\subsection{Influence of the inlet velocity profile}

Analysis performed in [6] provided an explanation for the mechanism leading to selfsustained oscillations and determined the conditions under which this phenomenon occurs. While the LES results obtained in [6] were in good qualitative agreement with the experimental findings, the quantitative discrepancies were significant and very puzzling. As can be seen in Fig. 6 the location of the local maximum of fluctuations obtained from LES is $x / D \approx 2$ while in the experiment it was found at a distance of $x / D \approx 4$ [6]. In the experiment, the height of this maximum was about $20 \%$ of $U_{0}$, i.e., almost two times larger than observed in LES. Additionally, in the experiment the self-sustained regime was observed also for larger $T i$ levels.

Taking into account the existence of the self-sustained regime, which was revealed to be very sensitive to the inlet parameters, it seemed reasonable to presume that the differences 
between the LES results and the measurements could have been caused by the inlet velocity profile. This supposition was additionally supported by the fact that the inlet profile in the experiment was different from the assumed HT profile. The measured velocity profile closely resembled the Blasius velocity profile. This motivated us to perform LES computations with the Blasius profile at the inlet. The following analysis is performed for two shear layer thicknesses: (i) $R / \theta=20$ which is below $R / \theta_{\text {crit }}$ and the self-sustained regime should not appear in this case; (ii) $R / \theta=28$ for which the self-sustained regime is expected as it was captured in the computations performed with the HT profile.

The results obtained for $R / \theta=20$ are presented in Fig. 7 showing the profiles of the time-averaged axial velocity and its fluctuations as a function of $T i$ level. It can be seen that when the Blasius profile is used the influence of the turbulence intensity is much more pronounced than in the results obtained with the HT profile (Cf. Fig. 4). Additionally, in Fig. 7 one may observe that the use of the Blasius velocity profile extends the potential core and slows down the growth in fluctuations compared to the results obtained with the HT profile. This is particularly well seen for the lowest $T i$ level. Figure 8 shows a comparison of fluctuating velocity profile in the logarithmic scale obtained for both velocity inlet profiles, $R / \theta=20$ and $T i=10^{-4}$. It is well seen that the application of the Blasius profile as a velocity inlet profile leads to significantly slower growth in perturbation. Moreover, it is shown that the fluctuating velocity profile for the case of the Blasius profile is characterized

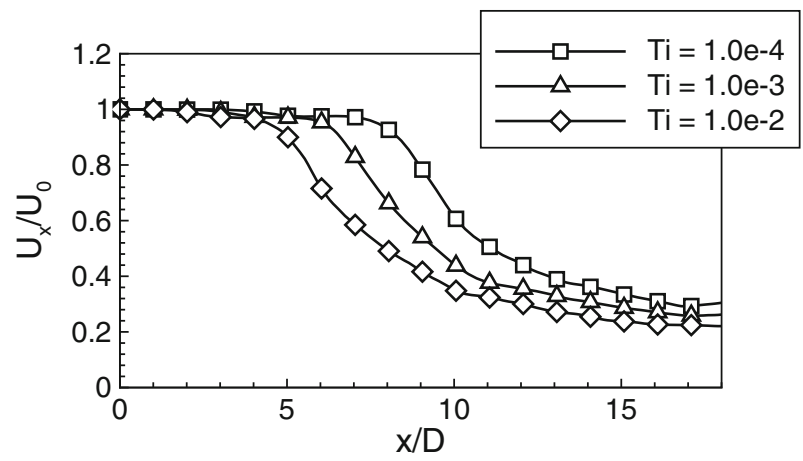

(a)

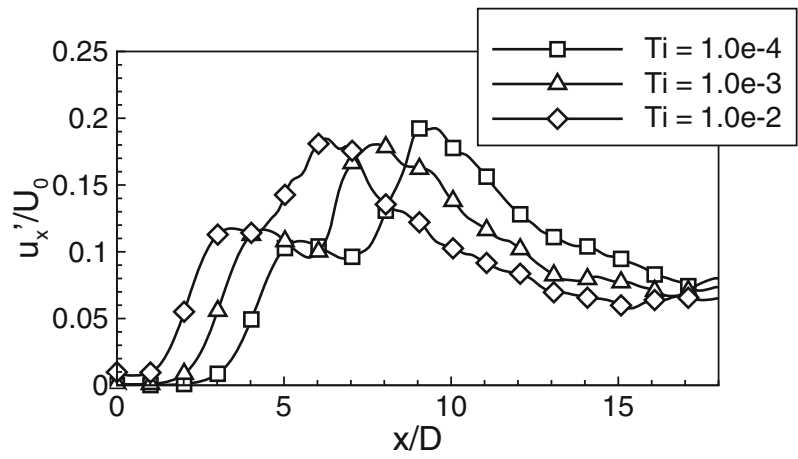

(b)

Fig. 7 Profiles of the time-averaged axial velocity (a) and its fluctuations (b) along the jet axis. Results obtained with the Blasius velocity profile with $R / \theta=20$ 


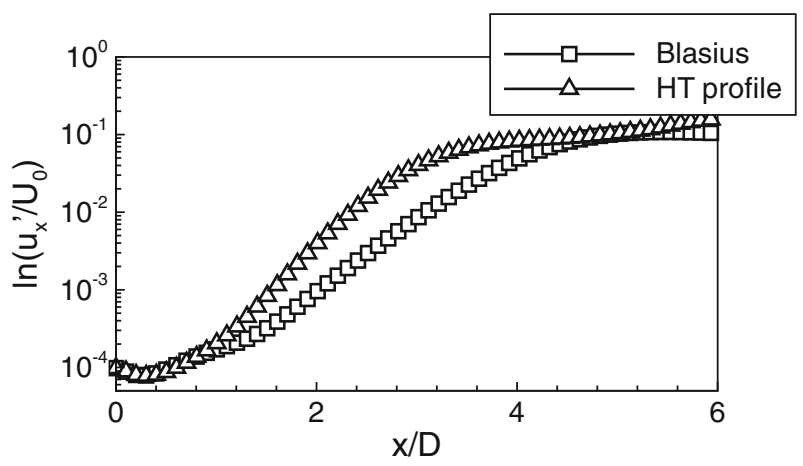

Fig. 8 Comparison of fluctuating velocity profile in logarithimc scale for both velocity inlet profile, $R / \theta=$ 20 and $T i=10^{-4}$

by linear growth at a distance $x / D=1.5-4$ while for the case of the HT profile this interval is shorter $x / D=1.5-3$. It is important also that linear growth in perturbation, for the case of the Blasius profile, is characterized also by a smaller slope than in the case of the HT profile.

Despite the significant differences between the results obtained for the various $T i$ levels none of the solutions for $R / \theta=20$ shows behaviour typical for the self-sustained regime. Although, in between $x / D=3-7$ the profiles of fluctuations in Fig. $7 \mathrm{~b}$ exhibit small local maxima, their shapes are significantly different from those observed in Fig. 5b for $R / \theta=28$ and $T i=10^{-4}$. There, the strong wavy behaviour of the profiles (fluctuation growth $\rightarrow$ maximum $\rightarrow$ minimum) was easily identified, here, in the range $x / D=3-7$ they are rather flat.

The situation changes completely when the Blasius velocity profile is used with the thinner momentum thickness, i.e., with $R / \theta=28$. The results obtained from these simulations are presented in Fig. 9, where, additionally, the results from the measurements [6] are presented. It can be seen that the profiles of the velocity fluctuations obtained from LES univocally indicate the occurrence of the self-sustained regime. The local maxima are very strong and approximately equal to $20 \%$ of $U_{0}$ which compares reasonably well with the experimental data. Moreover, the location of the maxima is shifted to $x / D \approx 2.9$ which when compared to the solutions obtained using the HT velocity profile (Cf. Fig. 5b) is closer to the experiment $x / D \approx 4$. Finally, from Fig. $9 \mathrm{~b}$ it may be seen that the self-sustained regime appears not only for the case with $T i=10^{-4}$ but also in the computations with $T i=10^{-3}$. This observation is also consistent with the experimental findings and further confirms that the use of the Blasius velocity profile at the inlet makes the simulation closer to the experimental data.

\subsection{Analysis in the spectral space}

The self-sustained regime is characterised by strong oscillations of both the velocity and pressure fields [6]. Figure 10 shows an evolution of the amplitude spectrum of the axial velocity along the jet centerline for the case with $R / \theta=20$ and $T i=10^{-4}$ for both inlet velocity profiles. The spectra are presented versus the Strouhal number $S t_{D}=f D / U_{0}$ where $f$ is the frequency of harmonics of the velocity signal. From Fig. 10 it can be seen that distinct peaks cannot be identified. Rather a broadband distribution is observed which 


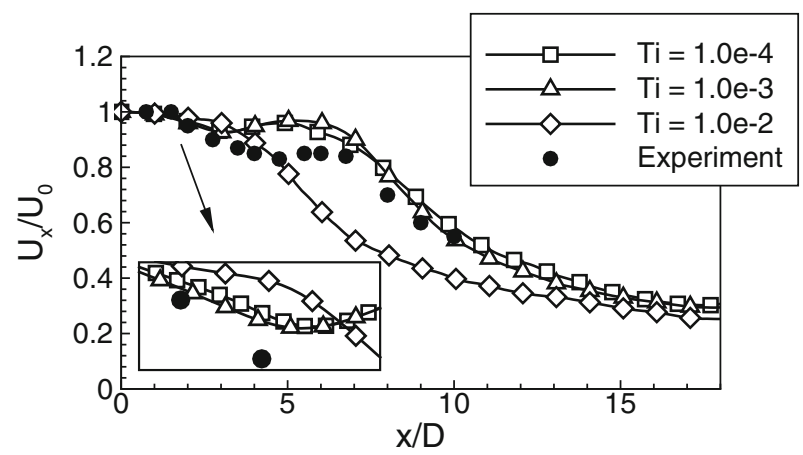

(a)

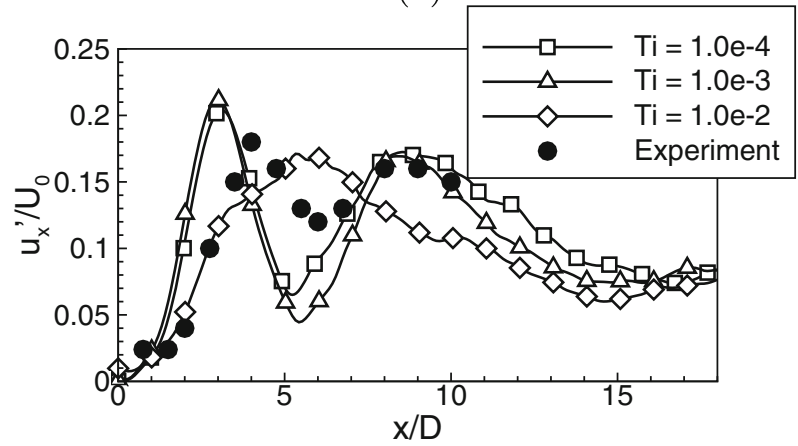

(b)

Fig. 9 Profiles of the time-averaged axial velocity (a) and its fluctuations (b) along the jet axis. Results obtained with the Blasius velocity profile with $R / \theta=28$

in the case of the HT profile is centered around $S t_{D} \approx 0.56$ corresponding well with the preferred mode frequency [10]. In addition the subharmonic at $S t_{D} \approx 0.3$ appears at a distance $x / D=4$ and attains its maximum at a distance $x / D=6$ which is characteristic for the pairing process of axisymmetric vortices. In the case of the Blasius profile the non-dimensional frequency of the most amplified mode is lower and characterized by $S t_{D}=0.51$ which also falls in the range of the preferred mode frequencies observed in the experiment. In this case the subharmonic is not observed.

Looking at the spectra in Fig. 11 obtained for $R / \theta=28$ a qualitative change of their shapes is evident. The peaks are sharper and significantly larger than the background fluctuations. Compared to the results shown in Fig. 10, presently the peaks related to the preferred modes are shifted towards larger frequencies. For the HT profile we have $S t_{D}=0.8$ with the subharmonic at $S t_{D}=0.4$, while in the case of the Blasius profile we observe $S t_{D}=0.75$ and the subharmonic at $S t_{D}=0.37$. It is necessary to note that in the experiment (see Fig. 4 in Ref. [6]) the preferred frequency corresponded to $S t_{D}=0.74$ with the subharmonic at $S t_{D}=0.37$. Hence, when the Blasius profile is used at the inlet the preferred frequencies agree almost perfectly. Taking into account the fact that the velocity profiles presented in the previous subsection obtained with the Blasius profile were also in good agreement with the experimental data we eventually conclude that the use of the Blasius profile as the inlet velocity is the correct choice for simulations of the self-sustained regime for the experimental configuration used in [6] . 

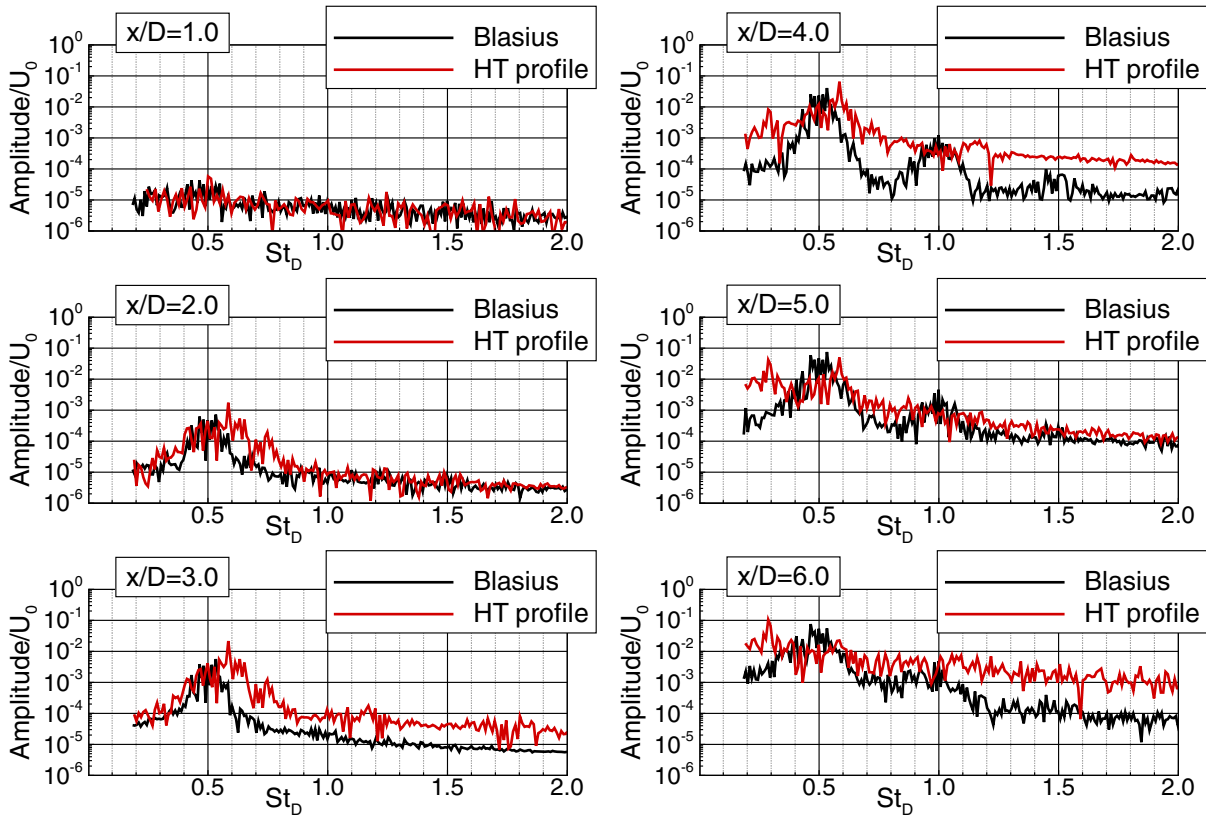

Fig. 10 Evolution of spectra along jet axis for $R / \theta=20, T i=10^{-4}$. Comparison of results obtained for both velocity profiles

To summarise this section it is important to stress the most important differences between the results obtained using the HT and Blasius inlet profiles. Starting from the case with the thicker shear layer for which the classical Kelvin-Helmholtz instability was detected, the Blasius profile leads to a longer potential core of the jet. It indicates that the perturbation growth in the near jet field is smaller compared with the HT inlet. Moreover, the characteristic oscillations frequency when the Blasius profile is used is significantly lower. Firstly, because the Blasius profile does not exhibit the inflexion point and consequently does not support the instability development right at the nozzle exit. In this case the inlet velocity profile evolves downstream and at a certain distance, due to shear stresses, transforms into the unstable velocity distribution. That means that the instability is triggered further downstream compared to the HT profile. Secondly, it was assumed that the momentum thickness of both velocity profiles is the same. However, as shown by linear stability theory, KelvinHelmholtz instability in the round jet scales on $R / \theta$-parameter, where $R$ stands for the radius where the inflexion point is located. In the case of the HT profile this radius is equal to the nozzle radius. In the case of the unstable velocity profile arising from the Blasius profile the inflexion point is located at a smaller radius. Consequently, the assumption of the Blasius velocity condition leads to a $R / \theta$-parameter smaller than in the case of the HT profile. As Kelvin-Helmholtz instability scales on $R / \theta$, in the case of Blasius velocity profile one may expect a smaller spatial perturbation growth rate and smaller characteristic frequency. This was confirmed by the numerical simulation results shown in Fig. 8, where the perturbation growth is shown, and in Fig. 10 presenting the spectral distributions. The linear stability theory can justify the differences in the solutions obtained with the Blasius and HT velocity profiles in the case of Kelvin-Helmholtz instability. In the case of synchronised 
oscillations triggered by the self-sustained mechanism such reasoning cannot be applied since large amplitude oscillations are observed right from the nozzle exit and the linear perturbation growth is not observed [6]. In the case of the Blasius velocity profile, as explained above, the Kelvin-Helmholtz instability develops further downstream from the nozzle. As a result the large vortices formed by the synchronised mode are established also further from the nozzle exit. The larger distance from the inlet allows for undisturbed growth of stronger vortices than in the case of the HT profile. Another interesting issue is the influence of the inlet velocity profile on the frequency shift of synchronised oscillations. The frequency selection mechanism of self-sustained oscillations is not fully understood, but it was stated in [6] that the frequency does not scale on $R / \theta$. Hence, it seems that the numerically predicted shift of the frequency caused by a change in the inlet profile is a consequence of the larger distance from the nozzle where the vortices are formed. It should be stressed that the influence of the velocity profile on the frequency of the synchronised oscillations, shown in Fig. 11, is much weaker than the influence observed for Kelvin-Helmholtz instability (see Fig. 10). However, a complete understanding of the frequency selection mechanism and its governing parameters for convective self-sustained oscillation needs further study.

\subsection{D flow behaviour}

The larger distance at which the instability develops when the Blasius profile is assumed allows for the forming of strong vortices which then lead to velocity fluctuations with large local maxima, as could be seen in Fig. 9, of the order of $20 \%$ of $U_{0}$. The vortices created near the inlet and also in the downstream region are visualised in Fig. 12 showing
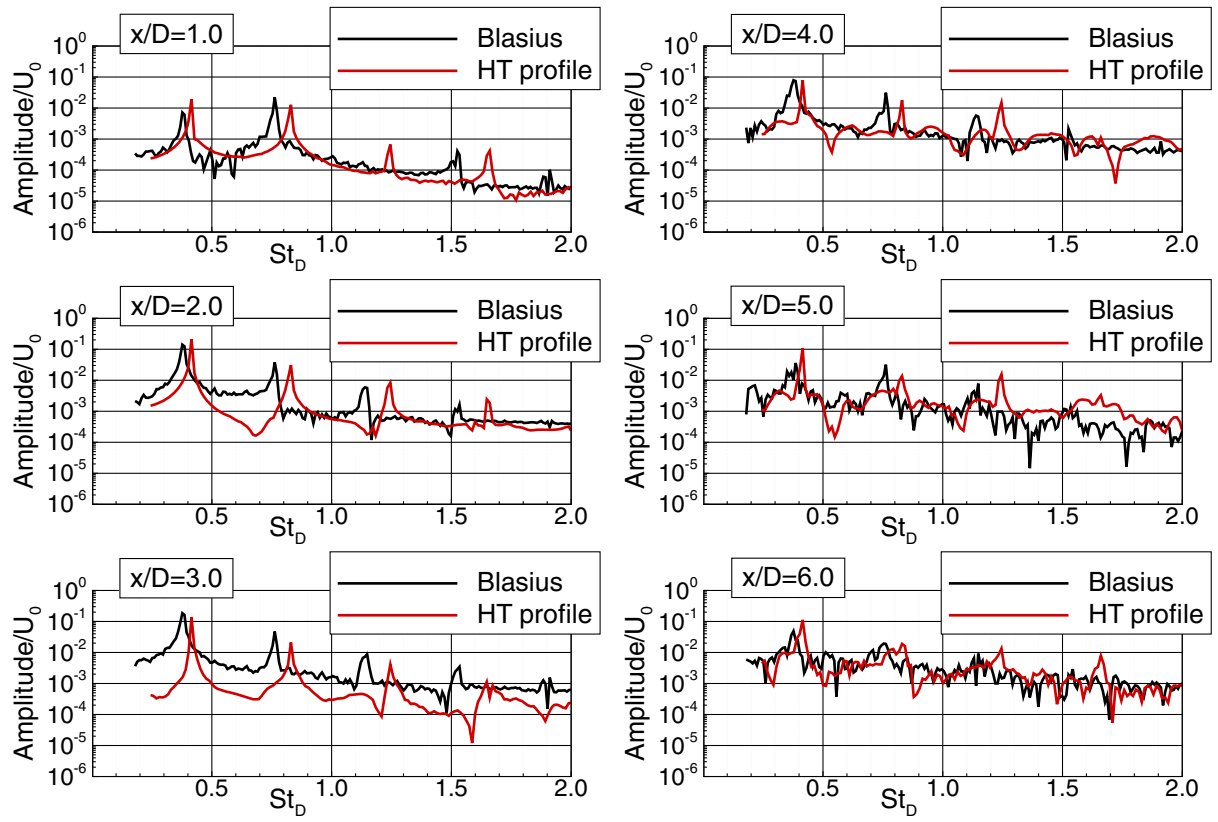

Fig. 11 Evolution of spectra along jet axis for $R / \theta=28, T i=10^{-4}$. Comparison of results obtained for both velocity profiles 


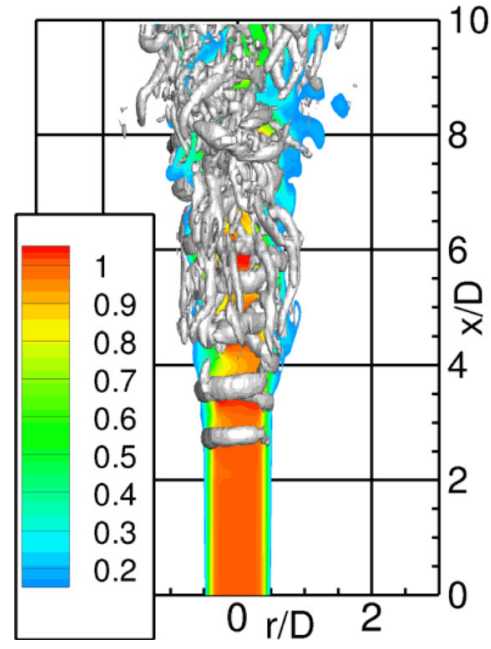

(a) $R / \theta=20$,

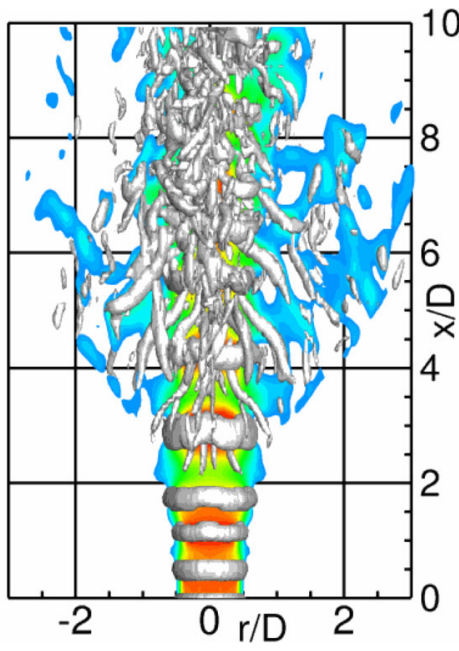

(b) $R / \theta=28$,

Fig. 12 Isosurfaces of $\mathrm{Q}$ - parameter $\left(Q=1\left(U_{0} / D\right)^{2}\right)$ and contours of the axial velocity in the main crosssection plane. Results obtained for using the Blasius velocity profile with (a) $R / \theta=20$ and (b) $R / \theta=28$, $T i=10^{-4}$

an isosurface of the Q-parameter equal to $Q=1\left(U_{0} / D\right)^{2}$. The Q-parameter is defined as $Q=\frac{1}{2}\left(S_{i j} S_{i j}-\Omega_{i j} \Omega_{i j}\right)$ where $S_{i j}$ and $\Omega_{i j}$ are symmetric and antisymmetric parts of the velocity gradient tensor. In Fig. 12 the Q-parameter exhibits toroidal structures resulting from the jet instability. The results are presented for two shear layer thicknesses with $R / \theta=20$ and $R / \theta=28$. It can be seen that in the latter case, when the critical thickness is exceeded, the formation of strong vortex structures is observed near the inlet plane, which consequently pair at a distance $x / D \approx 2.9$. The vortices are axisymmetric and almost not disturbed as the turbulence intensity is very low, $T i=10^{-4}$. The location where the vortices pair corresponds exactly to the local extrema of the mean and fluctuating velocity profiles (Cf. Fig. 9).

In the case when the HT profile is used at the inlet the vortical structures are not as strong and well formed as in Fig. 12, though they have a very similar shape. In effect the oscillations generated in the self-sustained regime when the HT profile is used are much weaker than in the case with the Blasius profile at the inlet. Most likely this results from the fact that in the former case the self-sustained oscillations are triggered close to the inlet plane, which may lead to some interactions with the flow in direct proximity to the inlet thus damping the oscillations intensity. On the other hand, when the Blasius profile is used and the self-sustained regime is triggered further from the inlet plane these interactions are weaker allowing for undisturbed growth of the vortex rings.

\subsection{Influence of subgrid model}

The importance of sub-grid modelling in shear layer type flows was demonstrated by Vreman et al. [27] based on a time evolution of coherent structures in a mixing layer. However, there are no studies concerning strictly round jet type flows where the turbulence develops 
initially in the shear layer region. Rather, there is a common belief that the LES results are insensitive to the sub-grid model used. This statement is generally true if we consider a far field region of fully developed turbulent flow where the sub-grid model participates mainly in the dissipation of energy. In a near field region the situation seems to be more complicated. Here, the level of sub-grid viscosity originating from the sub-grid model may be of significant importance. A lack of it may result in an unphysically unstable jet behaviour while its excess may damp developing turbulence. This is particularly true when the turbulence intensity at the nozzle exit is low. As shown in [23] in such cases even subtle differences in a numerical method may have a large impact on the results. Taking into account the sensitivity of the self-sustained regime to the inlet parameters one could suspect that in the performed simulations the role of the sub-grid model was not negligible. Hence, in this section we verify the influence of the sub-grid model on the results of simulations when the jets undergo the classical instability mechanism and when they are in the self-sustained regime. However, it should be stressed that the aim of this research is not to assess which sub-grid model performs the best for the analysed problem. This would require extensive comparisons with detailed experimental data and parametric studies including various mesh densities, turbulence intensities, inlet profiles, etc. Instead we try to determine the flow regime in which the choice of the sub-grid model is of crucial importance and the regime where the sub-grid model plays only a minor role. In particular, we are interested in how the prediction of the self-sustained regimes depends on the sub-grid model.

It was shown in the previous sections that the occurrence of the self-sustained regime is conditioned by two factors $-R / \theta$ ratio and turbulence intensity $T i$ - and is more pronounced for the Blasius profile at the inlet. In this section we analyse the cases with $R / \theta=20$ when the jets undergo the classical instability mechanism regardless of $T i$ level (Cf. Fig. 7), and with $R / \theta=28$ when the jet behaviour (classical instability $\longleftrightarrow$ self-sustained regime) changes depending on the $T i$ level (Cf. Fig. 9). In the performed simulations we only consider the Blasius profile as the inlet mean velocity profile. We compare the results obtained using four sub-grid models: (i) the filtered structure function (abbreviated as FSF) model proposed by Ducros et al. [16]; (ii) the dynamic Smagorinsky [28], referred to as the Germano model; (iii) the model proposed by Vreman [29]; (iv) the Smagorinsky model [30].

Figure 13 shows the contours of the instantaneous values of the normalised sub-grid viscosity $\left(v_{s g s} / v\right)$ in the main cross-section plane obtained in the simulations with $R / \theta=20$

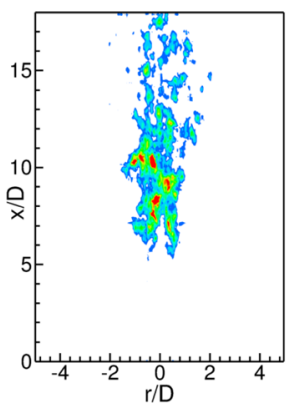

(a)

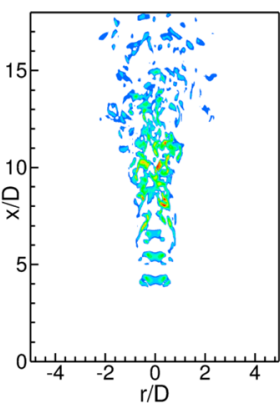

(b)

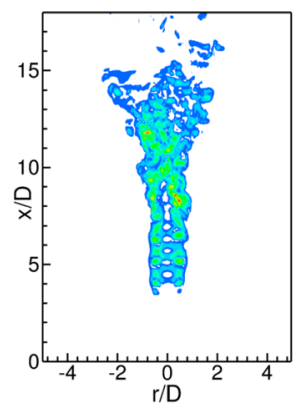

(c)

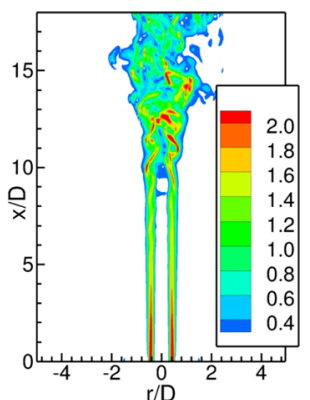

(d)

Fig. 13 Contours of an instantaneous sub-grid viscosity normalised by molecular viscosity. Results for shear layer characterized by $R / \theta=20, T i=10^{-4}$ obtained in the computations with the following sub-grid models: (a) FSF; (b) Germano; (c) Vreman; (d) Smagorinsky 
and $T i=10^{-4}$ for all the analysed sub-grid models. It is readily seen that the Smagorinsky model gives significantly different results than the other models. The Smagorinsky model has a strongly dissipative character, which is reflected here by the large values of the subgrid viscosity already appearing at the inlet and extending downstream along the shear layer. Because of such a distribution of the sub-grid viscosity the small scale phenomena produced by the shear stresses are damped. In effect, in jets with low $T i$ levels at the inlet the evolution of large scale structures is moved downstream and the growth in the perturbation is slowed down.

This is confirmed in Fig. 14 showing the profiles of the time-averaged axial velocity and its fluctuation along the jet axis for the cases with $T i=10^{-4}, 10^{-3}, 10^{-2}$. The results obtained with the FSF, Germano and Vreman model are very similar to each other. On the other hand, when the Smagorinsky model is used it is seen that for $T i=10^{-4}$ and $T i=10^{-3}$ the mean velocity profile is characterized by a considerably longer potential core compared to the results obtained with the remaining models. Additionally, the profile of the
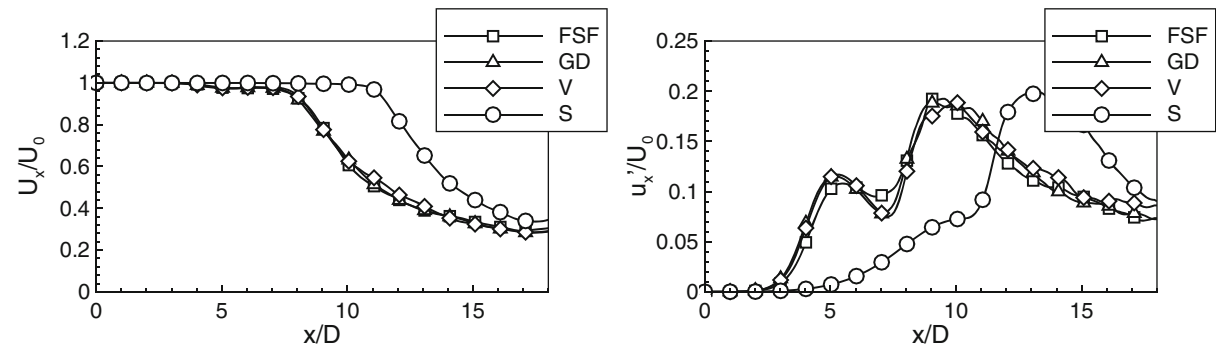

(a)
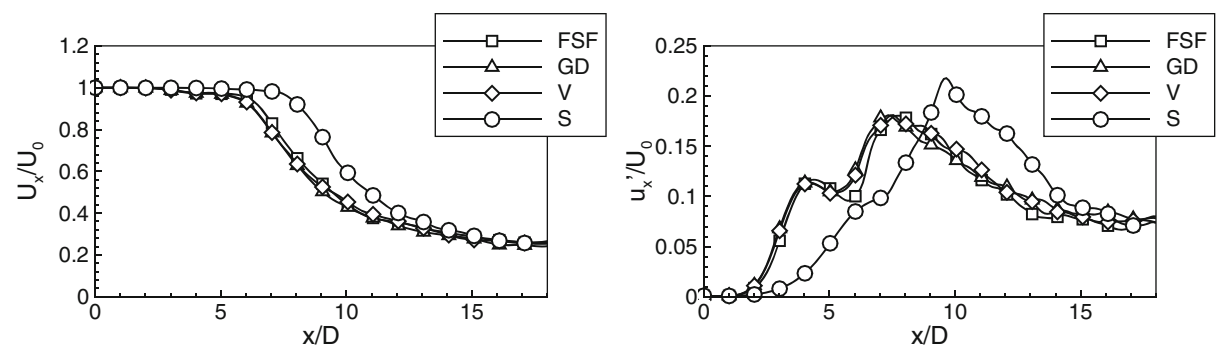

(b)
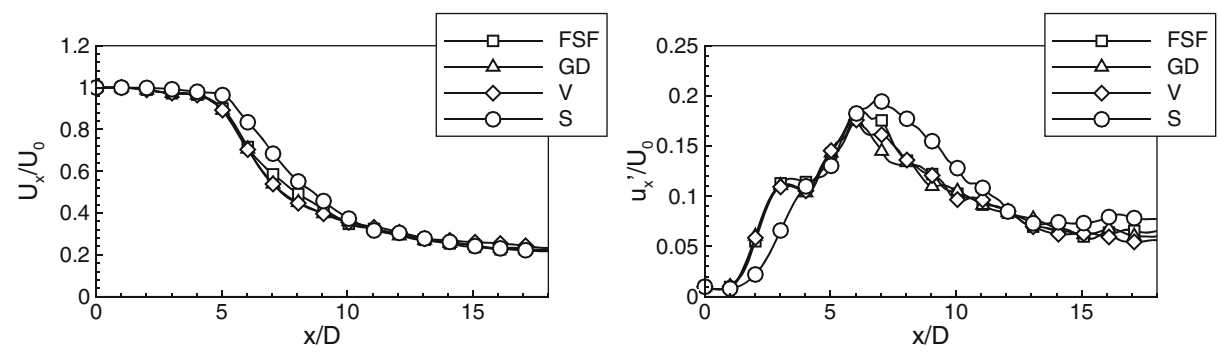

(c)

Fig. 14 Mean and fluctuating axial velocity profiles along the jet axis for shear layer characterized by $R / \theta=20$, (a) $T i=10^{-4}$, (b) $T i=10^{-3}$, (c) $T i=10^{-2}$. Comparison of results obtained using four sub-grid models: FSF, Germano denoted as 'GD', Vreman - 'V', Smagorinsky - 'S' 
velocity fluctuations obtained with the Smagorinsky model shows much slower growth in the perturbations. When the turbulent intensity rises to $T i=10^{-2}$ the differences between the results become smaller. This seems to confirm the fact that in the simulations of a jet the role of the sub-grid modelling is small when the turbulence intensity is large and dominates the flow dynamics at the sub-grid scale. In contrast, when the turbulence intensity is small the distribution of the sub-grid viscosity is a key factor.

The results of computations for $R / \theta=28$ presented in Fig. 9 showed that the selfsustained regime occurs for $T i=10^{-4}$ and $T i=10^{-3}$ while the turbulence intensity at a level of $T i=10^{-2}$ appeared to be sufficiently large to prevent the occurrence of this regime. However, to be sure that the comparison of the sub-grid models is performed for both the self-sustained and classical instability regimes we additionally consider the case with an even larger turbulence intensity level, $T i=5 \times 10^{-2}$.

Figure 15 shows the isosurfaces and contours of the instantaneous values of the Qparameter and the axial velocity obtained in the simulations for $R / \theta=28$ with $T i=10^{-4}$ with the four sub-grid models. It can be seen that the results obtained with the FSF, Germano and Vreman models are very similar whereas the solution obtained using the Smagorinsky model distinctly diverges from them. Similar discrepancies are revealed in the contours of the time-averaged results (not shown in the paper). We conclude that the Smagorinsky model, due to its excessive dissipative properties, is not suitable for the modelling of jets with low turbulence intensities, as it shifts the laminar-turbulent transition downstream. Hence, further analysis is confined to the FSF, Germano and Vreman models only. A comparison of the profiles of the time-averaged axial velocity is shown in Fig. 16. As before, as an indicator of the occurrence of the self-sustained regime we consider the local lowering of the mean velocity in the region of the $x / D \approx 3$ and the appearance of double maxima in the profiles of the fluctuations. Based on the presented results it turns out that in the cases with $T i=10^{-4}$ and $T i=5 \times 10^{-2}$ the results are virtually independent of the sub-grid model and the differences are small and only quantitative. From this observation one may conclude that when the inlet turbulence level is such that it leads to a well developed selfsustained regime or to the classical instability mechanism the role of the sub-grid modelling is small. The sensitivity of the results to the sub-grid model is only clearly revealed for the case with $T i=10^{-2}$ for which the velocity profiles are shown in Fig. 16c. This case seems to be a borderline case in which differences between the results are evident and where the

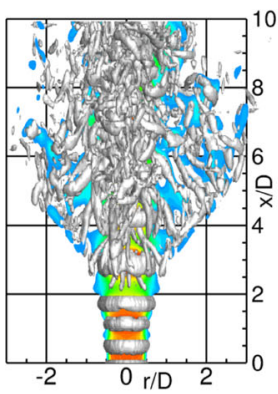

(a)

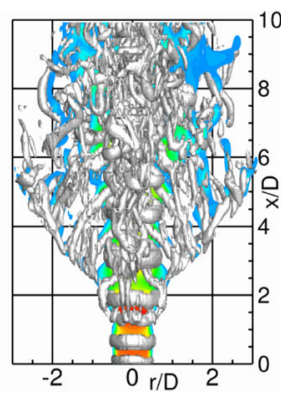

(b)

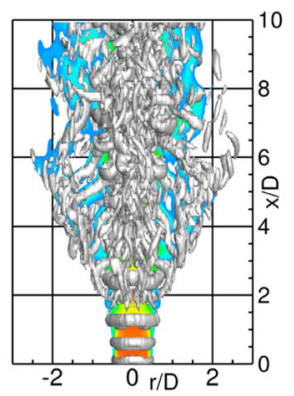

(c)

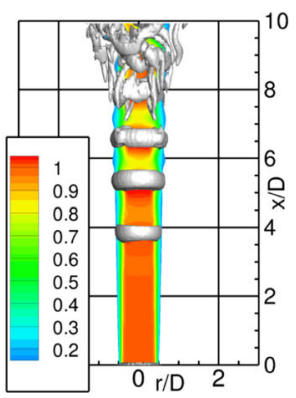

(d)

Fig. 15 Isosurfaces of $\mathrm{Q}$ - parameter $\left(Q=0.3\left(U_{0} / D\right)^{2}\right)$ and contours of the axial velocity in the main cross-section plane. Results obtained for using the Blasius velocity profile with $R / \theta=28, T i=10^{-4}$, (a) FSF, (b) Germano, (c) Vreman, (d) Smagorinsky models 

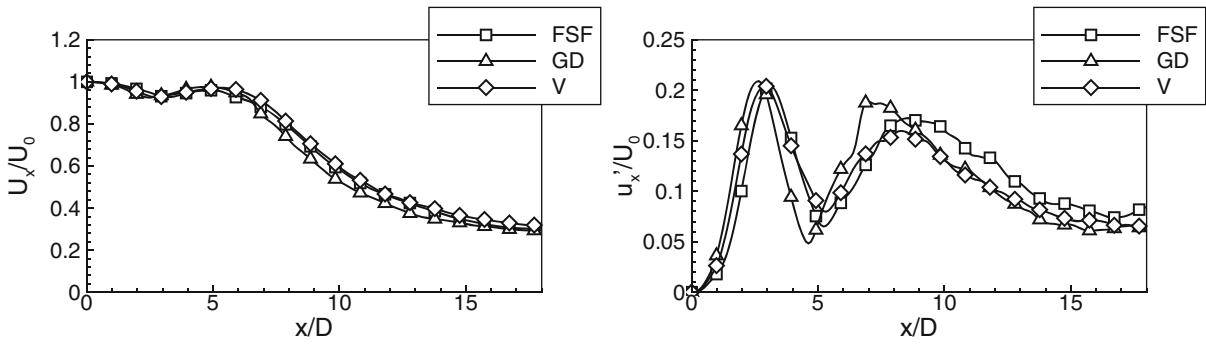

(a)

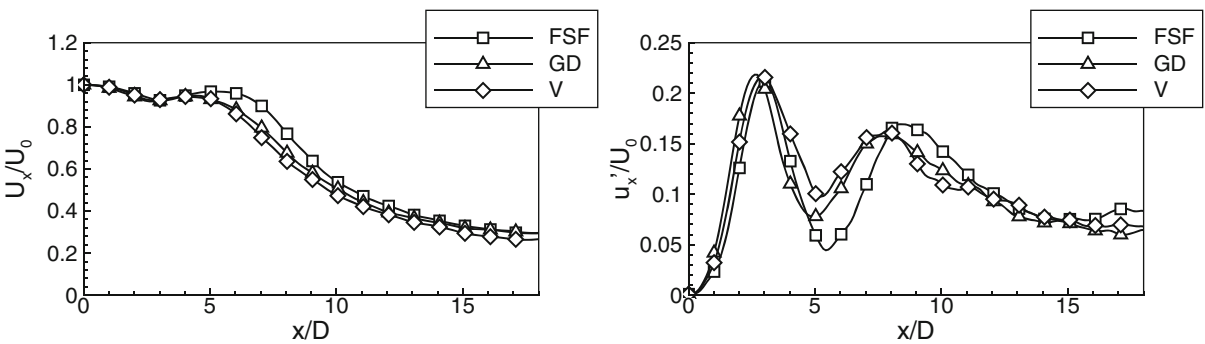

(b)
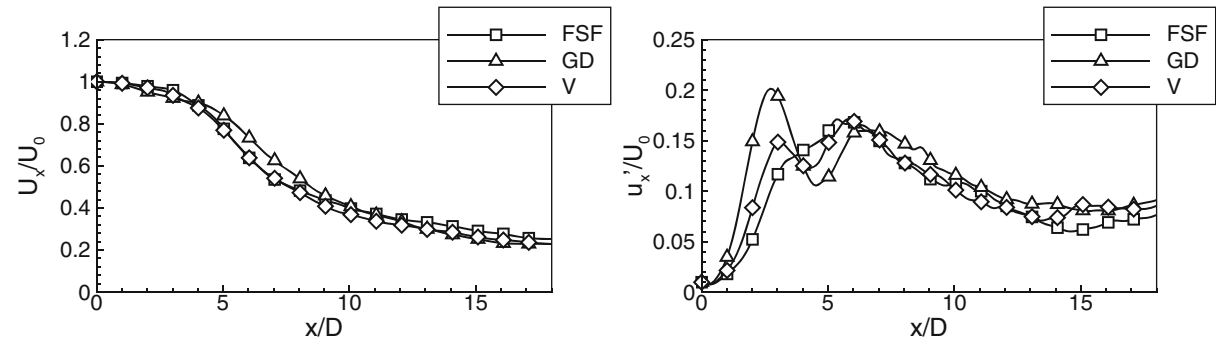

(c)
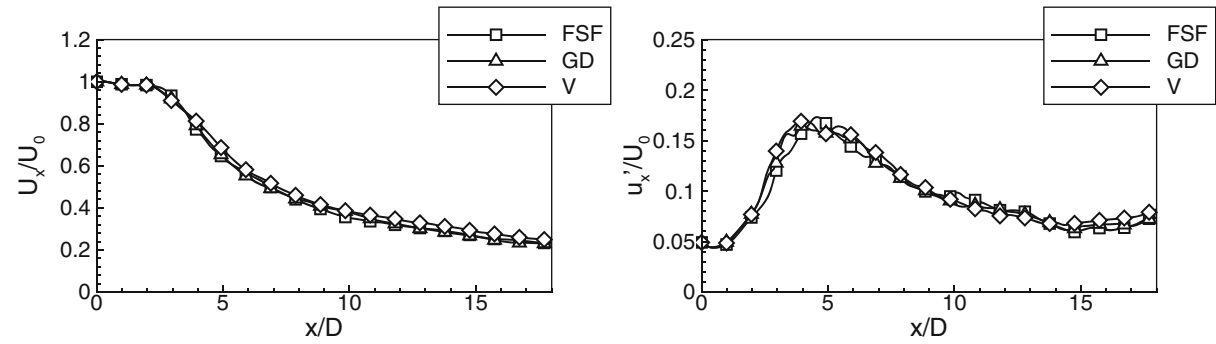

(d)

Fig. 16 Mean and fluctuating axial velocity profiles along the jet axis for shear layer characterized by $R / \theta=28$, (a) $T i=10^{-4}$, (b) $T i=10^{-3}$, (c) $T i=10^{-2}$, (d) $T i=5 \times 10^{-2}$. Comparison of results obtained for various sub-grid models

occurrence of the self-sustained regime was predicted only by the Germano model. The obtained results seem to confirm a general opinion that the choice of sub-grid model plays a crucial role in the transitional conitions while it becomes less important for well defined flow regimes. 


\section{Conclusion}

The paper presents a detailed analysis of LES predictions of a round jet under the selfsustained regime reported previously by Boguslawski et al. [6] on the basis of experimental and numerical results. There, a mechanism of self-sustained oscillations and the conditions under which this type of instability can be triggered were described. However, the LES results obtained in [6] were not in full agreement with the experimental data, and the observed discrepancies were left unexplained. The present studies aimed to analyse the possible sources of these discrepancies. The main attention was devoted to the analysis of the influence of the inlet velocity profile on the instability development. In [6] the hyperbolictangent profile was prescribed at the inlet, as done in many numerical studies of jet flows. However, in reality in the case of jets issuing from a high-contraction nozzle, as used in [6], the boundary layer is laminar and the velocity profile corresponds to the Blasius profile rather than the hyperbolic-tangent one. In this paper, we compared the results obtained using both profiles. It was shown that subtle differences in the inlet velocity profile lead to quite significant changes in the flow field in the near field. The application of the Blasius velocity profile leads to much a higher fluctuations level resulting from the self-sustained regime. Moreover, in this case we found that the peak of the fluctuating velocity moves further downstream from the inlet. Hence, we conclude that the results obtained with the Blasius profile are closer to the experiment than those obtained using the hyperbolic-tangent profile. However, it should be stressed that there are still some discrepancies between the LES results and the measurements. As suggested by an anonymous reviewer of this paper, it is most likely that the observed differences are caused by neglecting the region upstream to the inlet in the simulations, i.e., the nozzle. In reality, the oscillations occurring in the selfsustained regime are large and they can disturb the flow inside the nozzle. In consequence, the region immediately downstream of the nozzle can be affected by these disturbances.

In the last section of the paper we compared the results obtained using four well known sub-grid models: the filtered structure function model, the dynamic and classical Smagorinsky models and the model proposed by Vreman. Taking into account the sensitivity of the self-sustained regime to small differences in the inlet conditions one could assume that the sub-grid model may also play an important role. Comparative computations were performed for two shear layer thicknesses $R / \theta=20$ and $R / \theta=28$ with turbulence intensities $T i=1 \times 10^{-4}-5 \times 10^{-2}$. It was found that the classical Smagorinsky model is not suitable to simulate a jet with low $T i$. The excess of dissipation introduced by the Smagorinsky model dampens the growth of disturbances and shifts the laminar-turbulent transition far downstream. The results obtained for the case with $R / \theta=20$ applying the filtered structure function model, the dynamic Smagorinsky and Vreman models, are very similar. In the case of simulations with $R / \theta=28$ the results are very close to each other only when the turbulence intensity is very small $\left(T i=1 \times 10^{-4}\right)$ and the self-sustained regime is observed or when the turbulence intensity is large $\left(T i=5 \times 10^{-2}\right)$ and the classical instability mechanism occurs. On the other hand, in the transitional regimes the differences between the results are clearly visible. A lack of detailed experimental results does not allow us to point out which sub-grid model provides the most accurate solution. Nevertheless, an important conclusion resulting from the presented analysis is that the sub-grid modelling in simulations of jets being in transient conditions between the classical and self-sustained instability regimes certainly needs further study.

Acknowledgements The research was supported by Polish National Science Centre, project no. DEC2011/03/B/ST8/06401. The computations were carried out using PL-Grid Infrastructure. 
Open Access This article is distributed under the terms of the Creative Commons Attribution 4.0 International License (http://creativecommons.org/licenses/by/4.0/), which permits unrestricted use, distribution, and reproduction in any medium, provided you give appropriate credit to the original author(s) and the source, provide a link to the Creative Commons license, and indicate if changes were made.

\section{References}

1. Crow, S.C., Champage, F.H.: Orderly structure in jet turbulence. J. Fluid Mech. 48, 547-492 (1971)

2. Brown, G.L.: A.Roshko, On density effects and large structure in turbulent mixing layers. J. Fluid Mech. 64, 775-816 (1974)

3. Ball, C.G., Fellouah, H., Pollard, A.: The flow field in turbulent round free jets, Prog. Aerosp. Sci. 50, 1-26 (2012)

4. Hussain, A.K.M.F.: Coherent structures turbulence. J. Fluid Mech. 44, 1349-1357 (1986)

5. Fiedler, H.E.: Coherent structures in turbulent flows, Prog. Aerosp. Sci. 173, 303-356 (1988)

6. Boguslawski, A., Tyliszczak, A., Drobniak, S., Asendrych, D.: Self-sustained oscillations in homogeneous-density round jet. J. Turbul. 14, 25-52 (2013)

7. Lesshafft, L., Huerre, P., Sagaut, P.: Frequency selection in globally unstable round jets. Phys. Fluids 19, 25-52 (2007)

8. Mi, J., Xu, M., Zhou, T.: Reynolds number influence on statistical behaviors of turbulence in a circular free jet, Phys. Fluids, 25, 075101-1-30 (2013)

9. Meneveau, C., Katz, J.: Scale-invariance and turbulence models for large-eddy simulation. Annu. Rev. Fluid Mech. 32, 1-32 (2000)

10. Gutmark, E., Ho, C.M.: Preferred modes and the spreading rates of jets. Phys. Fluids 26, 2932-2938 (1983)

11. Fletcher, C.A.J.: Computational Techniques for Fluid Dynamics. Springer-Verlag, Berlin (1991)

12. Kyle, D.M., Sreenivansan, K.R.: The instability and breakdown of a round variable density jet. J. Fluid Mech. 249, 619-664 (1993)

13. Geurts, B.: Element of Direct and Large Eddy Simulations. Edwards, Philadelphia (2004)

14. Lesieur, M., Metais, O., Comte, P.: Large-Eddy Simulations of Turbulence. Cambrige University Pres, New York (2005)

15. Sagaut, P.: Large-Eddy Simulations for Incompressible Fows: An Introduction. Springer-Verlag, Berlin Heidelberg (2001)

16. Ducros, F., Comte, P., Lesieur, M.: Large-eddy simulation of transition to turbulence in a boundary layer developing spatially over a flat plate. J. Fluid Mech. 326, 1-36 (1996)

17. da Silva, C., Metais, O.: Vortex control of bifurcating jets. Phys. Fluids 14(11), 3798-3819 (2002)

18. Kempf, A., Klein, M., Janicka, J.: Efficient generation of initial- and inflow-conditions for transient flows in arbitrary geometry. Flow Turbul. Combust. 74, 67-84 (2005)

19. Williamson, J.H.: Low-storage Runge-Kutta schemes. J. Comput. Phys. 35, 48-56 (1980)

20. Lele, S.K.: Compact finite differences with spectral-like resolution. J. Comput. Phys. 103, 16-42 (1992)

21. Canuto, C., Hussaini, M.Y., Quarteroni, A., Zang, T.A.: Spectral Methods in Fluids Dynamic. SpringerVerlag, Berlin Heidelberg (1988)

22. Carpenter, M.H., Gottlieb, D., Abarbanel, S.: The stability of numerical boundary treatments for compact high order finite difference schemes. J. Comput. Phys. 108, 272-295 (1993)

23. Tyliszczak, A., Boguslawski, A., Drobniak, S.: Quality of LES predictions of isothermal and hot round jets, in Quality and Reliability of Large-Eddy Simulations Mayer, J., Geurts, B., Sagaut, P. (eds.) Springer Science + Buisiness Media B.V (2008)

24. Kuban, L., Laval, J.-P., Elsner, W., Tyliszczak, A.: LES modeling of converging-diverging turbulent channel flow. J. Turbul. 13, 1-19 (2012)

25. Aniszewski, W., Boguslawski, A., Marek, M.: A, Tyliszczak, A new approach to sub-grid surface tension for LES of two-phase flows. J. Comput. Phys. 231, 7368-7397 (2012)

26. Schmid, P.J., Henningson, D.S.: Stability and Transition in Shear Flows. Springer-Verlag, New York (2001)

27. Vreman, B., Geurts, B., Kuerten, H.: Large eddy simulation of the turbulent mixing layer. J. Fluid Mech. 339, 357-390 (1997)

28. Germano, M., Piomelli, U., Moin, P., Cabot, W.H.: Dynamic Subgrid-scale Eddy viscosity model, A. Phys. Fluids 3, 1760-1765 (1991) 
29. Vreman, A.W.: An eddy-viscosity subgrid-scale model for turbulent shear flow: Algebraic theory and applications. Phys. Fluids 16, 3670-3681 (2004)

30. Smagorinsky, J.: General circulation experiments with the primitive equations: I. The basic experiment. Mon. Weather Rev. 91, 99-164 (1963)

31. Monkewitz, P.A., Bechert, D.W., Barsikow, B., Lehmann, B.: Self- excited oscillations and mixing in a heated round jet. J. Fluid Mech. 213, 611-639 (1990) 